\title{
DiAgNOSTICS DANS LES PLASMAS DE DENSITÉ ET TEMPÉRATURE ÉLECTRONIQUES FAIBLES PAR MESURE D'IMPÉDANCE MUTUELLE (SONDE QUADRIPOLAIRE) ET D'IMPÉDANCE PROPRE (DIPÔLE DOUBLE SPHÊRE) $\left({ }^{*}\right)$
}

\author{
P. M. E. DECREAU et R. DEBRIE \\ C. R. P. E., Université d'Orléans, 45045 Orléans Cedex, France \\ (Reçu le 30 décembre 1976, accepté le 22 mars 1977)
}

\begin{abstract}
Résumé. - Nous avons mesuré, dans un plasma de simulation isotrope, l'impédance mutuelle complexe de sondes quadripolaires à configuration carrée ou linéaire, ainsi que l'admittance propre d'une sonde formée d'un dipôle double sphère.

Chacun de ces systèmes donne accès à une ou plusieurs déterminations de la densité et de la température électronique, que nous avons pu comparer entre elles.
\end{abstract}

\begin{abstract}
We have measured, in an isotropic plasma of simulation, the complex mutual impedance of quadrupole probes in square and linear configurations as well as the self impedance of a double sphere dipole probe.

Each of these devices leads to one or several determinations of the electronic density and temperature, that we were able to compare.
\end{abstract}

1. Introduction. - La sonde quadripolaire, imaginée par Storey [1], a fait l'objet de nombreuses études théoriques et développements expérimentaux, tant en laboratoire que dans le milieu spatial [2-9].

La réponse en fréquence de cette sonde présente une résonance à la fréquence plasma $f_{\mathrm{p}}$ suivies d'antirésonances dont le nombre et la fréquence permettent de déduire la température électionique [5].

D'autre part, la mesure de l'admittance propre d'une sonde formée d'un simple dipôle double sphère donne également la densité et la température électronique.

Nous avons étudié en caisson de simulation, dans le cas d'un plasma isotrope, le comportement de sondes quadripolaires (configuration carrée et linéaire) et d'une sonde double sphère. Les résultats obtenus permettent de comparer ces différents dispositifs et les utilisations que l'on peut en faire pour le diagnostic du plasma.

2. Les dispositifs de mesure. - La sonde quadripolaire est formée de deux dipôles double sphère, l'un émetteur, l'autre récepteur.

Classiquement, les électrodes sphériques de la sonde sont reliées électriquement aux systèmes d'émission et de réception, par l'intermédiaire de fortes impédances série, de manière telle que le courant d'émission

(*) Communication présentée à la Conférence Nationale de Physique des Plasmas, Paris, 6-10 décembre 1976. soit constant, et la tension de réception mesurée en circuit ouvert. On a ainsi accès directement à l'impédance de transfert entre le dipôle émetteur et le dipôle récepteur.

Nous avons utilisé ce système dans le cas d'une sonde quadripolaire de configuration carrée de $20 \mathrm{~cm}$ de côté. Les impédances séries et les préamplificateurs se trouvent dans un petit module, disposé au sommet d'un tétraèdre représenté schématiquement en figure 4 .

La liaison se fait par fils fins nus, de $15 \mathrm{~cm}$ de longueur, $50 \mu \mathrm{m}$ de diamètre, disposés dans de légers bras isolants. Les sphères sont pleines de rayons $1,5 \mathrm{~cm}$.

Par ailleurs, nous avons utilisé le dispositif représenté schématiquement en figure 1 ; l'émission se fait à tension constante, par l'intermédiaire de câbles coaxiaux arrivant jusqu'au niveau des sphères. Le courant injecté dans le plasma est mesuré indépendamment, à l'aide d'un tore entourant l'un des câbles d'émission. Ce dispositif, qui est utilisé pour des mesures similaires sur le satellite GEOS, est décrit en [10]. Les deux sphères d'émission représentent ainsi la sonde dipôle double sphère. Cette sonde, complétée d'un dipôle récepteur, forme une sonde quadripolaire à configuration linéaire, qui nous donne en pratique la référence des paramètres du plasma. Les quatre sphères sont placées à égale distance $(8 \mathrm{~cm})$ les unes des autre. Elles sont soit pleines, soit grillagées, de rayon $1 \mathrm{~cm}$. Chaque sphère de réception est reliée, par l'intermédiaire de fils fins ( $6 \mathrm{~cm}$ de longueur, $25 \mu \mathrm{m}$ de diamètre), 


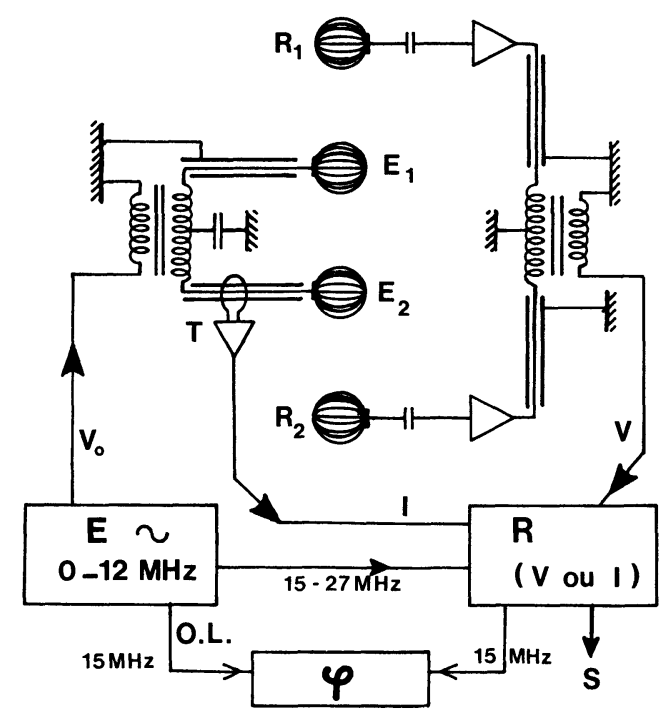

FIG. 1. - Dispositif expérimental. Les sphères d'émission $E_{1} E_{2}$ sont alimentées en opposition de phase par le générateur à fréquence variable $E$. Le courant émis $I$ est mesuré à l'aide du tore $\mathrm{T}$. La différence de potentiel $V$ entre les sphères de réception $R_{1}$ et $R_{2}$ est transmise au système d'analyse $R$, par l'intermédiaire de fortes impédances séries (capacités de $0,1 \mathrm{pF}$ ). La mesure des amplitudes des signaux reçus est donnée, après détection, par la sortie S. Leurs déphasages par rapport au signal émis $V_{0}$ est mesuré par l'appareil $\varphi(\mathrm{H}$. P. type $3575 \mathrm{~A})$.

[Experimental device. The emitting spheres, $E_{1} E_{2}$, are supplied by the generator $E$. The emitted current $I$ is measured with the core $\mathrm{T}$. The potential difference $V$, between the receiving spheres $\mathbf{R}_{1} \mathbf{R}_{2}$, is transmitted to the analysing system $R$ through large series impedances (capacities of $0.1 \mathrm{pF}$ ). The amplitude of the received signals is detected and available at the output $S$. The phase difference between a received signal and the emitted signal $V_{0}$, is measured through the device $\varphi($ H. P. type $3575 \mathrm{~A})$.]

à une forte impédance série (faible capacité) et au préamplificateur miniaturisé placés dans un blindage, ce dernier étant glissé à l'intérieur du bras isolant qui porte la sphère.

Le système électronique représenté schématiquement en figure 1 a été utilisé pour faire la mesure des courbes de réponse des sondes à configuration linéaire (sondes dipolaire et quadripolaire de la figure 1). C'est le même ensemble d'analyse (émission et réception) qui a été utilisé pour les mesures concernant la sonde quadripolaire carrée à courant d'émission constant décrite auparavant.

Les plasmas dans lesquels nous avons fait nos mesures, sont des plasmas de type ionosphérique, produits dans la chambre de simulation du C. R.P. E. décrite en [11].

3. Théorie. - Les travaux théoriques de Chasseriaux et Odéro [7] ont montré que l'influence mutuelle entre deux sphères d'un dipôle jous un rôle fondamental dans l'expression de son admittance propre. Pour aborder ce problème dans le cas de grilles sphériques en plasma chaud isotrope, ils ont utilisé un modèle multiple Water Bag pour approximer la fonction de distribution maxwellienne des vitesses. Ils montrent que les résultats sont indépendants de la répartition des charges sur les sphères, si l'on néglige les termes d'ordre quatre en $\eta=R / L, R$ étant le rayon d'une sphère, et $L$ la distance entre sphères, valeurs normalisées à la longueur de Debye.

Dans le cadre de cette approximation, tout à fait légitime dans notre cas, ces auteurs donnent l'expression de l'impédance propre d'une sphère isolée $Z_{\mathrm{s}}$ (réf. [7], p. 279) et de l'impédance mutuelle entre deux sphères, $Z_{\mathrm{i}}(L)$ (référence [7], p. 283).

De plus, en considérant la contribution des différentes charges pulsantes à l'émission, aux potentiels atteints par les différentes sphères de la sonde, ils obtiennent l'expression de l'impédance propre du dipôle double sphère :

$$
Z_{\mathrm{d}}=2\left[Z_{\mathrm{s}}-Z_{\mathrm{i}}(L)\right]
$$

et de l'impédance mutuelle de la quadripolaire, soit :

$$
Z_{\mathrm{m}}=2\left[Z_{\mathrm{i}}(L)-Z_{\mathrm{i}}(2 L)\right]
$$

en configuration linéaire, où $L$ est la distance normalisée entre deux sphères consécutives, et :

$$
Z_{\mathrm{m}}=2\left[Z_{\mathrm{i}}(L)-Z_{\mathrm{i}}(\sqrt{2} L)\right]
$$

en configuration carrée, où $L$ représente le côté normalisé du carré.

Nous avons appliqué ces calculs au cas où l'approximation du circuit de réception ouvert n'est plus valable. En effet, l'impédance propre des sphères de réception (capacités de $1 \mathrm{pF}$ dans le vide), n'est pas toujours très faible devant celle des capacités séries présentes entre chacune de ces sphères et la masse $\left(C_{\mathrm{R}}=0,1 \mathrm{pF}\right)$, notamment au voisinage de la fréquence plasma, ou l'impédance propre d'une sphère isolée tend vers l'infini.

Dans ces conditions, les charges présentes sur les sphères de réception ne sont plus négligeables et il faut considérer la sonde quadripolaire sans l'aspect de quatre conducteurs chargés en présence les uns des autres. Connaissant les expressions de l'impédance propre d'une sphère, et mutuelle entre deux sphères, la résolution de ce problème est simple, et l'impédance mutuelle obtenue pour une sonde quadripolaire est $Z_{\mathrm{m}_{1}}$ telle que :

$$
Z_{\mathrm{m}_{1}}=Z_{\mathrm{m}} /\left[1+i \omega C_{\mathrm{R}} Z_{\mathrm{dr}} / 2\right]
$$

où $Z_{\mathrm{dr}}$ est l'impédance propre du dipôle de réception considéré seul, et $Z_{\mathrm{m}}$ l'impédance mutuelle en circuit de réception ouvert (expression (3) ou (4)).

De plus, dans le cas d'une sonde quadripolaire classique, où le courant est injecté dans le plasma à travers une faible capacité $C_{\mathrm{E}}$, cette dernière ne présente pas toujours une impédance très grande devant celle du dipôle d'émission lui-même et le courant émis n'est pas toujours constant. On montre que l'impédance que l'on mesure dans ce cas est $Z_{\mathrm{m}_{2}}$ telle que :

$$
\begin{array}{r}
Z_{\mathrm{m}_{2}}=Z_{\mathrm{m}} /\left[\left(1+i \omega C_{\mathrm{R}} Z_{\mathrm{dr}} / 2\right)\right. \\
+\left(1+i \omega C_{\mathrm{E}} Z_{\mathrm{de}} / 2\right)+ \\
\left.+\omega^{2} C_{\mathrm{E}} C_{\mathrm{R}} Z_{\mathrm{m}}^{2} / 4\right]
\end{array}
$$


où $Z_{\mathrm{de}}$ est l'impédance propre du dipôle d'émission considéré seul. $Z_{\mathrm{m}_{2}}$ est le rapport de la tension reçue, au courant de référence $i \omega C_{\mathrm{E}} V_{\mathrm{E}}, 2 V_{\mathrm{E}}$ étant la tension d'émission.

L'influence des capacités $C_{\mathrm{E}}$ et $C_{\mathrm{R}}$ se fait surtout sentir au voisinage de la fréquence plasma, où les quantités $Z_{\mathrm{dr}}, Z_{\mathrm{de}}, Z_{\mathrm{m}}$ sont maxima. On peut constater ce fait dans le cas de la figure 5, où l'on compare des courbes de réponse données par les expressions (3) et (5).

4. Les résultats. - Nous prendrons comme référence les valeurs des paramètres du plasma, obtenues par une méthode classique, décrite par exemple dans [5], et qui utilise la réponse en module de la sonde quadripolaire : la fréquence plasma $f_{\mathrm{p}}$ est celle de la résonance, la longueur de Debye $\lambda_{\mathrm{D}}$ (qui conduit à la température électronique $T_{\mathrm{e}}$ ) est déduite à partir de la position des creux observés aux fréquences supérieures à $f_{\mathbf{p}}$.

Cette mesure de $T_{\mathrm{e}}$ a d'ailleurs été comparée avec celle donnée par une sonde de Langmuir très élaborée, dans le caisson dont nous disposons [6], et les résultats obtenus étaient identiques, à la précision de mesure près $(5 \%)$.

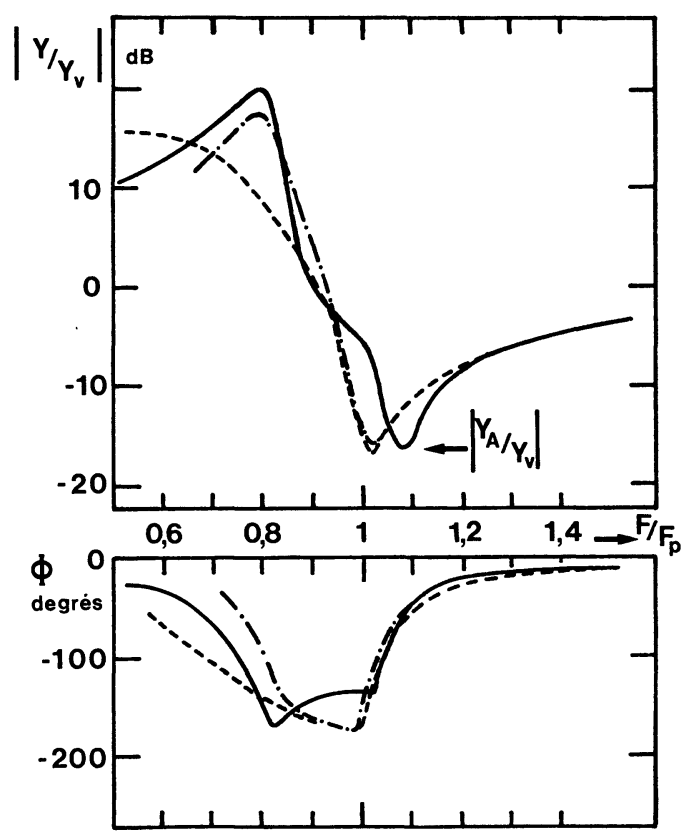

Fig. 2. - Dipôle double sphère. Admittance propre normalisée par rapport à sa valeur dans le vide, $Y / Y_{\mathrm{v}}$, en fonction de la fréquence $f$ normalisée à la fréquence plasma $f_{\mathrm{p}}$. On donne, dans la représentation module - $|Y| Y_{\mathrm{v}} \mid$ - et argument - $\Phi$ - les trois courbes suivantes : - expérimentale ; ---- théorique suivant l'expression $(3) ;-\ldots$. . théorique en présence d'une gaine vide d'épaisseur $1 \lambda_{\mathrm{D}}$; la distance entre sphères est $D=20 \lambda_{\mathrm{D}}$. [Double sphere dipole. Self admittance normalized with respect to its value in vacuo $Y / Y_{\mathrm{v}}$, as a function of the frequency $f$, normalized with respect to the plasma frequency $f_{\mathrm{p}}$. The following curves are drawn in the modulus $-|Y| Y_{\mathrm{v}} \mid$ - and phase - $\Phi$ - representation : - experimental curve ; --- theoretical curve following the expression $(3) ;-.-$. theoreitcal curve in presence of an empty sheath of thickness $1 \lambda_{\mathrm{D}}$. The distance between spheres is $D=20 \lambda_{\mathrm{D}}$.]
Ayant précisé nos valeurs de référence pour $f_{\mathrm{p}}$ et $\lambda_{\mathrm{D}}$, nous pouvons discuter plus en détail les résultats présentés sur les figures 2 à 6 .

Dans le cas du dipôle double sphère (Fig. 2), l'écart entre les courbes de réponse expérimentales et les courbes théoriques correspondant à l'expression (3) peut s'expliquer en partie par la présence de gaines ioniques, qui modifient de manière importante la valeur de l'impédance propre d'une sphère isolée $Z_{\mathrm{s}}$. Cette valeur est prépondérante dans l'expression (3), sauf au voisinage de $f_{\mathrm{p}}$, où la présence des gaînes devient négligeable si $L \gg R$ [7]. On constate effectivement (Fig. 2) qu'à l'antirésonance, proche de $f_{\mathrm{p}}$, l'admittance du dipôle, est très peu affectée par la présence d'une gaine vide d'électrons. Le module de cette antirésonance donne dans ces conditions accès à une mesure valable de la température. Nous avons pu tester cette méthode de mesure pour un domaine de variation de $L$ compris entre 5 et 30 (Fig. 3).

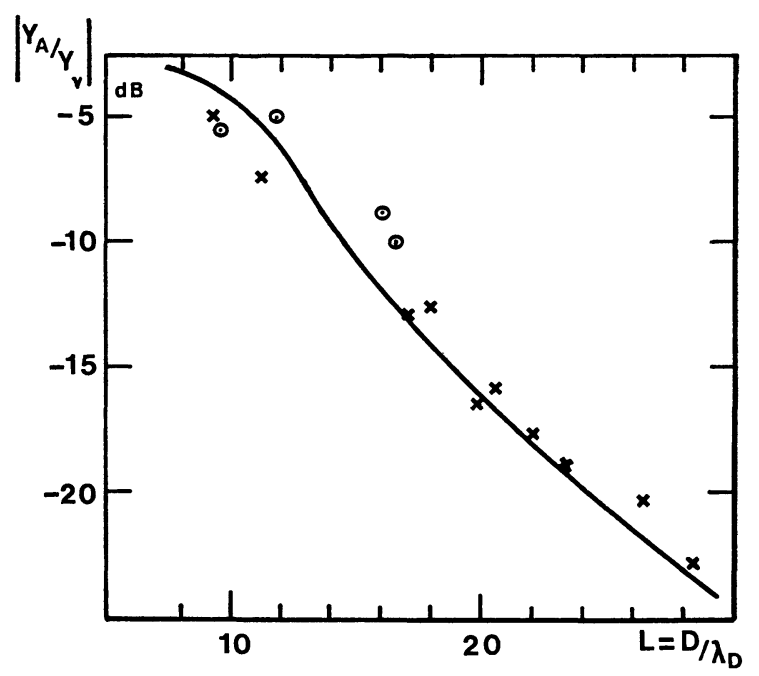

Fig. 3. - Dipôle double sphère. Module de l'admittance propre à l'antirésonance, $\left|Y_{\mathrm{a}} / Y_{\mathrm{v}}\right|$, en fonction de $D / \lambda_{\mathrm{D}}$, distance entre sphères normalisée à la longueur de Debye. Par rapport à la courbe théorique, on donne les valeurs obtenues espérimentalement, le paramètre $L=D / \lambda_{\mathrm{D}}$ étant évalué à partir de la mesure en sonde quadripolaire faite simultanément. On a utilisé pour le dipôle émissif des sphères solides - symboles (o) ainsi que des sphères grillagées - symboles $(+)-$.

[Double sphree dipole. Modulus of the self admittance at the antiresonance, $\left|Y_{\mathrm{a}} / Y_{\mathrm{v}}\right|$, as a function of $D / \lambda_{\mathrm{D}}$, normalized distance between spheres. Beside the theoretical curve, one gives the values obtained experimentaly, the parameter $L=D / \lambda_{\mathrm{D}}$ being evaluated through the quadrupole probe measurements made simultaneously. The emitting dipole was constituted either of solid spheres - symbols (o) - or of meshed spheres - symbols $(+)$.]

Les résultats expérimentaux montrent que la mesure de la température électronique est possible avec une précision de $\pm 30 \%$ environ $( \pm 15 \%$ sur la longueur de Debye).

Par ailleurs, la fréquence plasma donnée par le dipôle double sphère est systématiquement supérieure 
à celle donnée par mutuelle. Cet écart, que nous n'expliquons pas de manière satisfaisante, reste faible (2 à $12 \%$ ) sur de nombreuses mesures, et nous évaluons la précision sur la mesure de densité par sonde double sphère à $\pm 10 \%$ en se référant à un écart systématique de $+7 \%$ sur la fréquence plasma.

Dans le cas de la sonde quadripolaire, l'amplitude de la résonance est inférieure à sa valeur théorique (Fig. 4 et 5). Diverses causes de ce phénomène peuvent être évoquées : présences physiques des sphères et de leurs gaînes, des fils fins, des supports, des parois du caisson, etc... Nous ne discuterons pas ce point ici.

En revanche, nous avons envisagé l'utilisation de la réponse en argument de la sonde quadripolaire, comme mesure des paramètres du plasma. On peut en effet constater (Fig. 5) que la courbe de réponse expérimentale est assez proche de la valeur théorique attendue. La fréquence plasma est moins bien définie que dans la réponse en module : le saut de phase entre $180^{\circ}$ et $0^{\circ}$ se fait de manière plus arrondie que la théorie ne la prévoit. Cependant, les positions en fréquence des zéros de

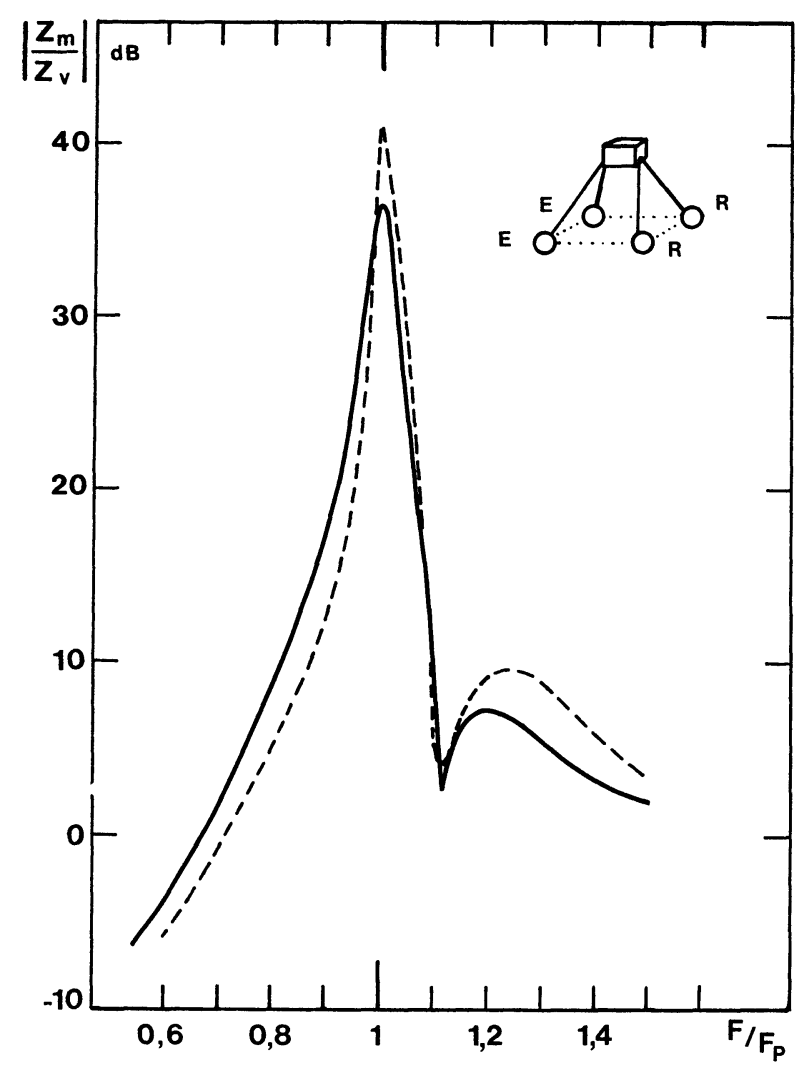

Fig. 4. - Sonde quadripolaire carrée. Module de l'impédance mutuelle normalisée à sa valeur dans le vide, $\left|Z_{\mathrm{m}}\right| Z_{\mathrm{v}} \mid$, en fonction de la fréquence normalisée $f / f_{\mathrm{p}}$. A côté de la courbe expérimentale (-) on a représenté la courbe théorique (---) dans le cas de quatre sphères isolées dans l'espace; la distance entre sphères est $D=22 \lambda_{\mathrm{D}}$.

[Quadrupole probe in square configuration. Modulus of the mutual impedance normalized with respect to its value in vacuo, $\left|Z_{\mathrm{m}} / Z_{\mathrm{v}}\right|$, as a function of the normalized frequency, $f \mid f_{\mathrm{p}}$. Beside the experimental curve (-), the theoretical one (---) is drawn in the case of four spheres isolated in space. The distance between spheres is $D=22 \lambda_{\mathrm{D}}$.]

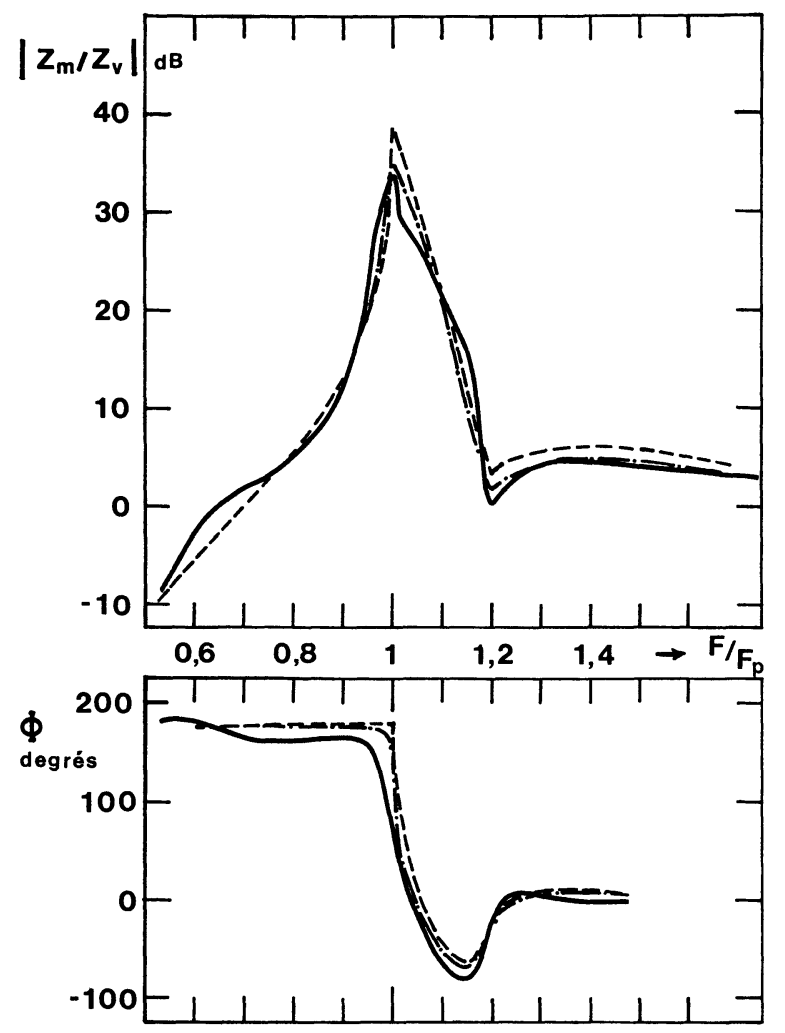

FIG. 5. - Sonde quadripolaire en ligne. Impédance mutuelle normalisée par rapport à sa valeur dans le vide, $Z_{\mathrm{m}} / Z_{\mathrm{v}}$, en fonction de la fréquence normalisée, $f / f_{\mathrm{p}}$. On donne dans la représentation module $-\left|Z_{\mathrm{m}}\right| Z_{\mathrm{v}} \mid$ - et argument $-\Phi$-, les courbes suivantes : - expérimentale (dispositif de la Fig. 1) ; ------ théorique dans le cas de quatre sphères isolées dans l'espace ; - . - ; théorique dans le cas où l'on tient compte des liaisons électriques : les impédances série à la réception sont deux éléments en parallèle, une capacité de $0,1 \mathrm{pF}$ et une résistance de $10 \mathrm{M} \Omega$; la distance entre sphères est $D=16 \lambda_{\mathrm{D}}$.

[Quadrupole probe in linear configuration. Mutual impedance normalized with respect to its value in vacuo, $Z_{\mathrm{m}} / Z_{\mathrm{v}}$ as a function of the normalized frequency, $f / f_{\mathrm{p}}$. The following curves are drawn in the modulus $-\left|Z_{\mathrm{m}} / Z_{\mathrm{v}}\right|-$ and phase $-\Phi-$ representation: - experimental curve (device of Fig. 1); ---- theoretical curve in the case of four spheres isolated in space; - . - . theoretical curve when taking into account electric connections : the series impedance at reception consists of two elements in parallel, a capacity of $0.1 \mathrm{pF}$ and a resistance of $10 \mathrm{M} \Omega$. The distance between spheres is $D=16 \lambda_{\mathrm{D}}$.]

phase correspondent assez bien aux valeurs attendues (Fig. 6).

En fait, les précisions de nos mesures en phases se sont trouvées assez mauvaises $\left(\sim \pm 20^{\circ}\right)$, pour des raisons contingentes. Nous pensons que des mesures de phases faites dans de bonnes conditions, devraient conduire à une précision en température meilleure que celle que l'on peut estimer à partir des courbes de la figure 6. Surtout, ces mesures conduisent à une évaluation du paramètre $L$ (par la position de $F \varphi 01$ ), même lorsque celui-ci est assez faible et que la courbe en module ne présente plus de creux au-dessus de la fréquence plasma, et par conséquent pas de mesure de $T_{\mathrm{e}}$ 

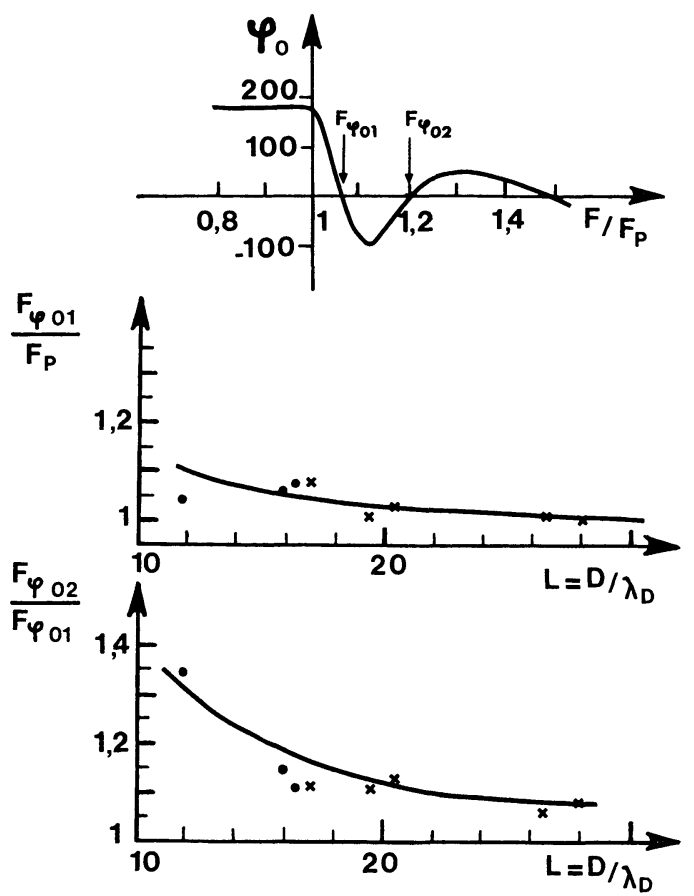

FIG. 6. - Sonde quadripolaire en ligne. Position en fréquence, des passages par zéro de l'argument $\varphi$ de l'impédance normalisée. A côté des courbes théoriques représentant les quantités $f \varphi \mathrm{o}_{1} / f_{\mathrm{p}}$ et $f \varphi \mathrm{o}_{2} / f \varphi \mathrm{o}_{1}$ en fonction du paramètre $L=D / \lambda_{\mathrm{D}}$ on donne les valeurs obtenues expérimentalement à l'aide du dispositif représenté en figure 1 . Les valeurs de $\mathrm{L}$ prises en référence sont obtenues à partir de la position en fréquence de l'antirésonance du module de l'impédance mutuelle. Les symboles (o) correspondent à des sphères d'émission solides et les symboles $(X)$ à des sphères grillagées.

[Quadrupole probe in linear configuration. The argument $\varphi$ of the normalized impedance comes to zero for several frequency values, $f \varphi$ oi. Besides the theoretical curves representing the quantities $f \varphi o_{1} / f_{\mathrm{p}}$ and $f \varphi \mathrm{o}_{2} / f \varphi \mathrm{o}_{1}$ as a function of the parameter $L=D / \lambda_{\mathrm{D}}$, one gives the values obtained experimentally with the device described in figure 1 . The $L$ values referred to are obtained through the frequency of the antiresonance of $\left|Z_{\mathrm{m}} / Z_{\mathrm{v}}\right|$. The symbols (o) refer to solid emitting spheres, and the symbols (x) to meshed spheres.] par la méthode classique que nous décrivions au début de ce paragraphe.

5. Conclusion. - Les différentes courbes de réponse obtenues dans un plasma isotrope permettent une comparaison quantitative de deux types de sonde. La mesure du module de l'impédance mutuelle d'une sonde quadripolaire permet une détermination précise $(<5 \%)$ de la densité et de la température électronique. Une autre détermination de la température est obtenue en complétant l'analyse par une mesure de l'argument de l'impédance mutuelle, cette dernière mesure pouvant en principe étendre le domaine d'utilisation de la sonde quadripolaire.

L'accord entre les courbes de réponse théoriques et expérimentales est comparable pour les deux configurations de sonde étudiées (Figs. 4 et 5). La configuration carrée présente cependant à priori l'avantage suivant: le plasma ne se trouve perturbé sur aucun trajet émetteur-récepteur, par la présence d'une troisième sphère de la sonde. Cette propriété n'est pas réalisée en configuration linéaire. En revanche, c'est dans ce dernier cas que l'interprétation des courbes de réponses en présence d'un champ magnétique faible sera la plus aisée.

Les mesures sur la simple sonde double sphère ont montré que, comme le prévoit la théorie, la gaine thermique intervient peu sur l'amplitude de l'impédance propre de la sonde au voisinage de la fréquence plasma. La mesure de cette amplitude donne ainsi une estimation de la température en accord à $\pm 30 \%$ près avec les mesures obtenues par sonde quadripolaire.

Remerciements. - Nous remercions M. Navet, qui a mis à notre disposition ses programmes de calcul sur le modèle multiple Water-Bag, ainsi que Y. Arnal pour sa grande compétence dans la production du plasma isotrope de grand volume que nous avons utilisé.

\section{Bibliographie}

[1] Storey, L. R. O., Aubry, M. P., Meyer, P. in plasma waves in Space and in the Laboratory, ed. J. O. Thomas and B. J. Landmark (Edinburgh Univ. Press) 1 (1969) 303.

[2] Grard, R. J. L., Alta Freq. 38 (1969) 97.

[3] Renard, C., Debrie, R., C. R. Hebd. Séan. Acad. Sci. B 270 (1970) 649.

[4] Rooy, B., Feix, M. R., Storey, L. R. O., Plasma Phys. 14 (1972) 275.

[5] Chasseriaux, J. M., Debrie, R., Renard, C., J. Plasma Phys. 8 (1972) 231.
[6] Chasseriaux, J. M., Thèse Paris VI (1973).

[7] Chasseriaux, J. M., Odero, D., J. Plasma Phys. 10 (1973) 265.

[8] Pottelette, R., Rooy, B., Fiala, V., J. Plasma Phys., 14 (1975) 209.

[9] Debrie, R., Arnal, Y., Illiano, J. M., Phys. Lett. 56 (1976) 95.

[10] Sene, F., Karczewski, J. F., Decreau, P. M. E. Note Technique C. R. P. E. n ${ }^{\circ} 26$, à paraître.

[11] Illiano, J. M., Arnal, Y., Gille, P., Colloque International du CNES, Paris, mai 1975. 